\title{
APPLICATIONS OF NONSTANDARD MODELS AND LEBESGUE MEASURE TO SEQUENCES OF NATURAL NUMBERS
}

\author{
STEVEN C. LETH
}

\begin{abstract}
By use of a nonstandard model, sequences of natural numbers are associated with a collection of closed subsets of reals in a natural way. The topological and measure-theoretic properties of the associated closed sets are used to prove standard theorems and define new density functions on sequences.
\end{abstract}

1. The main goal of this work is to continue to provide a framework for the use of nonstandard methods in combinatorial number theory which was begun in [Le]. Whereas only countable models were used in that paper we will concentrate here on those properties which require the use of a larger model, i.e. some degree of saturation or a large language. By results of Friedman $[\mathbf{K}]$ the use of countable models cannot lead to theorems whose standard proofs are set-theoretically more complicated than the nonstandard proofs, while Henson, Kaufmann and Keisler [HKK] show that standard proofs of results obtained using some saturation may require correspondingly more choice. In light of this it is perhaps not surprising that the standard theorems generated here seem considerably more difficult to prove in a classical way than those in [Le].

In $\S 2$ for each standard sequence $A$ and infinite number $z$ (i.e. $z \in{ }^{*} \mathbf{N}-\mathbf{N}$ ) we define "st $z(A)$ " to be the subset of $[0,1]$ obtained by dividing each element of * $A$ by $z$ and taking standard parts. Standard conditions on a sequence $A$ are given which are equivalent to "st ${ }_{z}(A)$ always contains an interval" and "st ${ }_{z}(A)$ never contains an interval." Using techniques similar to those used in [Le] some standard theorems involving these conditions are proved.

In $\S 3$ two new density functions for sequences are introduced by using the Lebesgue measure of the sets $\operatorname{st}_{z}(A)$. Various properties of these density functions are developed, including some similarities to the classical asymptotic and Schnirelmann densities. The density functions suggest a notion of a "partial basis" which is somewhat analogous to the classical notion of basis.

In $\S 4$ various sequence versions of the classical result of Steinhaus that the sum of two sets of real numbers of positive measure contains an interval are developed.

Finally, in $\S 5$, some problems and concluding remarks are given.

2. We wish to apply the measure theory of the real numbers to sequences by using a nonstandard model. In order to do so we will need a model which includes a symbol for each real number as well as for each sequence of real numbers, all functions from $\mathbf{R}^{n}$ into $\mathbf{R}$, and the symbol " $\varepsilon$ ". We could use a nonstandard

Received by the editors July 7, 1986 and, in revised form, May 14, 1987. This paper contains some of the results of the author's doctoral thesis (The University of Colorado, 1985).

1980 Mathematics Subject ('lassification (1985 Revision). Primary 11U10, 03H15, 11B05, 11 B75. 
universe as used in the construction of the Loeb measure [Lo], but we will not need as much structure as that.

We are mostly interested in the additive properties of unbounded sequences of natural numbers, and the classical development of this subject provides the motivation for much of the work done here. However, none of our results require that the sequences be whole numbers. Thus the sequences considered here will always be unbounded increasing sequences of reals unless otherwise specified. Our intended standard model is a set containing at least all subsets of the reals and all functions from $\mathbf{R}^{n}$ into $\mathbf{R}$.

For each infinite $z \in{ }^{*} \mathbf{N}$ we define a mapping taking standard sequences into subsets of $[0,1]$.

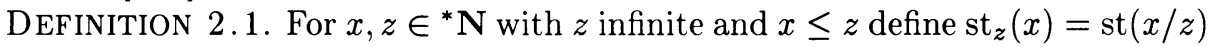
("st" means "standard part of").

For $A$ an infinite sequence and $z$ infinite in ${ }^{*} \mathbf{N}$ we write

$$
\operatorname{st}_{z}(A)=\left\{\operatorname{st}_{z}(a): a \in{ }^{*} A, a \leq z\right\} .
$$

Proposition 2.2. For any sequence $A$ and any infinite $z \in{ }^{*} \mathbf{N} \operatorname{st}_{z}(A)$ is closed.

PROOF. If $r \in[0,1]$ is a limit point of $\operatorname{st}_{z}(A)$ let $a$ be the nearest point of ${ }^{*} A$ to $[r z]$. Then $\operatorname{st}_{z}(a)=r$.

Proposition 2.2 is a special case of the well-known fact that the standard part of any internal set is closed, which is due to Robinson $[\mathbf{R}]$.

We ask the question: under what conditions does $\operatorname{st}_{z}(A)$ never contain an interval? In order to answer this question we need a notion which was introduced in [Le].

DEFinition 2.3. A sequence $A$ is said to be $e$-nowhere dense iff for any $\varepsilon>0$ there is a $\delta>0$ so that for any sufficiently large $n \in \mathbf{N}$ there is a gap in $A$ of size at least $\delta n$ between $n$ and $n+\varepsilon n$.

THEOREM 2.4. A sequence $A$ is e-nowhere dense iff for all $z \in{ }^{*} \mathbf{N}-\mathbf{N s t}_{\boldsymbol{z}}(A)$ is nowhere dense.

PROOF. $\Rightarrow$ Suppose that $\operatorname{st}_{z}(A)$ is not nowhere dense for some $z \in{ }^{*} \mathbf{N}-\mathbf{N}$. By Proposition 2.2 it must then contain an interval $[r, r+\varepsilon r], \varepsilon>0$. Then by transfer $A$ satisfies the sentence which asserts, for each $k$ :

"There are arbitrarily large numbers $n$ with all gaps in $A$ between $n$ and $n+\varepsilon n$ less than $n / k$ ".

Thus, no $\delta>0$ exists for this $\varepsilon$.

$\Leftarrow$ Suppose that there is an $\varepsilon>0$ with no such $\delta$.

By transfer we can then find an infinitesimal $\sigma>0$ and an infinite $n$ with no gaps bigger than $\delta n$ between $n$ and $n+\varepsilon n$. Letting $z=[n+\varepsilon n]$ we see that $\operatorname{st}_{z}(A)$ contains an interval.

Proposition 2.5. The sequence $A=\left\langle a_{n}\right\rangle$ has the property that $\operatorname{st}_{z}(A)=[0,1]$ for all $z \in{ }^{*} \mathbf{N}-\mathbf{N}$ iff $\lim _{n \rightarrow \infty} a_{n+1} / a_{n}=1$.

Proof. Clear.

We now want to find conditions equivalent to: $\operatorname{st}_{\boldsymbol{z}}(A)$ always contains an interval. The standard translation of this property is somewhat cumbersome. 
DEFINITION 2.6. We say that a sequence $A$ has the interval property iff for any infinite sequence $C \subseteq \mathbf{N}$ there is an $\varepsilon>0$ such that for all $\delta>0$ there are infinitely many $c \in C$ with an interval of length $\varepsilon c$ below them which contains no gap in $A$ of size $\delta c$.

In answer to a question posed by Kunen, Merrill has shown the following

THEOREM 2.7 (MERRILL). The interval property is complete $\Pi_{1}^{1}$ (for proof of this theorem see $[\mathbf{M}])$.

This shows that the definition given here cannot be simplified in an essential way. We see below, however, that from the nonstandard viewpoint the notion is natural.

THEOREM 2.8. A sequence $A$ has the interval property iff for every $z \in{ }^{*} \mathbf{N}$ $\operatorname{st}_{\boldsymbol{z}}(A)$ contains an interval.

ProOF. $\Rightarrow$ Suppose that there is an infinite $z \in{ }^{*} \mathbf{N}$ with no interval in $\operatorname{st}_{z}(A)$. Then for any positive standard integer $k$ there is a positive standard rational $\delta_{k}$ such that any interval in ${ }^{*} \mathrm{~N}$ of size $z / k$ below $z$ contains a gap in $A$ of $\operatorname{size} \delta z$. We transfer each of the sentences:

"There is a number $z \in \mathbf{N}, z>k$ such that for $i=1, \ldots, k$ every interval of size $z / i$ below $i$ contains a gap in $A$ of size $\delta_{i} z$." If $c_{k}$ is the realization in $\mathbf{N}$ of the $k$ th statement above then it is easy to see that $c=\left\langle c_{k}\right\rangle$ cannot have the required $\varepsilon>0$, so that $A$ cannot have the interval property.

$\Leftarrow$ Suppose now that there is an infinite $C \subseteq \mathbf{N}$ without the required property. Let $c$ be an infinite element of ${ }^{*} C$. By transfer, for each standard $\varepsilon>0$ there is a standard $\delta>0$ so that all intervals below $c$ of length at least $\varepsilon c$ contain a gap in $A$ of size $\delta c$. Thus $\operatorname{st}_{z}(A)$ is nowhere dense.

DEFInITION 2.9. The sequence $A$ is said to limit-separate $B$ iff for any $\varepsilon>0$ there is an $N$ so that if $\left[m_{1}, m_{2}\right]$ is an interval of natural numbers containing more than $N$ elements of $B$ and no elements of $A$ then $\left(m_{2}-m_{1}\right) / m_{1}<\varepsilon$.

Proposition 2.10. If $A$ limit-separates $B$ then for all $z \in{ }^{*} \mathbf{N}-\mathbf{N}$ any nonisolated point of $\operatorname{st}_{z}(B)$ is also an element of $\operatorname{st}_{z}(A)$.

PROOF. Let $x$ be a nonisolated point of $\operatorname{st}_{z}(B)$. Then for any standard $\varepsilon>0$ the interval $[(x-\varepsilon) z,(x+\varepsilon) z]$ contains infinitely many elements of $B$. Thus every such interval must contain elements of $A$, so that $x \in \operatorname{st}_{z}(A)$.

It is shown in [Le] that if $A_{i}$ limit separates $B_{i}$ for $i=1, \ldots, N$ and $\sum_{i=1}^{N} A_{i}$ is $e$-nowhere dense then $\sum_{i=1}^{N} B_{i}$ is $e$-nowhere dense. The following result is very similar.

THEOREM 2.11. Suppose that $A_{i}$ limit-separates $B_{i}$ for $i=1, \ldots, N$ and that $\sum_{i=1}^{N} B_{i}$ has the interval property. Then $\sum_{i=1}^{N} A_{i}$ also has the interval property.

ProOF. By induction on $N$. For $N=1$ the theorem is clear.

If the theorem is true up to $N$ we note that

$$
\operatorname{st}_{z}\left(\sum_{i=1}^{N+1} B_{i}\right)=\operatorname{st}_{z}\left(B_{1}\right)+\cdots+\operatorname{st}_{z}\left(B_{N+1}\right) \cap[0,1] .
$$


The above sum is contained in $\mathrm{st}_{z}\left(A_{1}\right)+\cdots+\mathrm{st}_{z}\left(A_{N+1}\right)$ except, perhaps, at isolated points of one of the $B_{i}$ by Proposition 2.10. Since there are only countably many such points, $\operatorname{st}_{z}\left(\sum_{i=1}^{N+1} B_{i}\right)-$ st $_{z}\left(\sum_{i=1}^{N+1} A_{i}\right)$ must be meager (by the induction hypothesis) and thus cannot contain an interval by the Baire Category Theorem. Since $\sum_{i=1}^{N+1} B_{i}$ has the interval property, st $_{z}\left(\sum_{i=1}^{N+1} B_{i}\right)$ does contain an interval, so that $\operatorname{st}_{z}\left(\sum_{i=1}^{N+1} A_{i}\right)$ must also.

THEOREM 2.12. Let $A$ be a sequence such that for some $z \in{ }^{*} \mathbf{N}-\mathbf{N s t}_{z}(A)$ contains only isolated points, and $B$ be e-nowhere dense. Then neither $A+B$ nor $k A$ (for $k$ any natural number) has the interval property.

Proof. Let $z$ be infinite in ${ }^{*} \mathbf{N}$ with $\operatorname{st}_{z}(A)$ isolated. Then $\operatorname{st}_{z}(A+B)$ is a countable union of nowhere dense sets and so is meager. Thus $\operatorname{st}_{z}(A+B)$ does not contain an interval and so $A+B$ cannot have the interval property. Similarly $s t_{z}(k A)$ is, in fact, countable and so also contains no interval.

3. We now turn our attention to measure. Since $\operatorname{st}_{z}(A)$ is always closed it is always measurable. We show below that there are notions of density based on the Lebesgue measure of $\operatorname{st}_{z}(A)$ which correspond in some ways to the classical notions of density which we now review.

DEFInITION 3.1. The Schnirelmann density, $\sigma$, of a sequence $A$ of natural numbers is given by $\sigma(A)=\inf _{n} A(n) / n$ where $A(n)$ is the number of elements of $A \leq n$. Similarly, the upper and lower asymptotic density, $\bar{d}$ and $\underline{d}$, are defined by

$$
\bar{d}(A)=\varlimsup_{n} A(n) / n, \quad \underline{d}(A)=\underline{\lim } A(n) / n .
$$

Definition 3.2. A sequence $A$ of natural numbers is said to be a basis iff there is a $k$ so that $k A$ (the $k$-fold set sum of $A$ ) is all of $\mathbf{N}$.

These notions of density and basis have been extensively studied and there are many deep theorems about them. We recall (without proof) two major results.

THEOREM 3.3 (MANN). For $A, B$ sequences of natural numbers with $0 \in A$, $0 \in B$,

$$
\sigma(A+B) \geq \min \left\{\begin{array}{l}
\sigma(A)+\sigma(B), \\
1 .
\end{array}\right.
$$

Corollary 3.4 (Schnirelmann). If $\sigma(A)>0$ and $0 \in A$ then $A$ is a basis.

We note that Corollary 3.4 is immediate from Theorem 3.3 since it is easy to see that if $\sigma(A)=1$ then $A=\mathbf{N}$. However, this result was proved by Schnirelmann before Mann's theorem. The special case of Mann's theorem in which $A=B$ was proved by Khintchin.

For proofs of these theorems (and many more results related to density and basis) see [HR].

We define below two new asymptotic densities by using the measure of $\operatorname{st}_{z}(A)$ (we use $m$ for Lebesgue measure).

DEFINITION 3.5. Let $A$ be an unbounded increasing sequence of reals and define

$$
\begin{aligned}
& \underline{\mu}(A)=\inf _{z \in *^{*}-\mathbf{N}} m\left(\operatorname{st}_{z}(A)\right), \\
& \bar{\mu}(A)=\sup _{z \in \cdot \mathbf{N}-\mathbf{N}} m\left(\text { st }_{z}(A)\right) .
\end{aligned}
$$


These functions would not, of course, be very interesting if they depended on which nonstandard model was being used. We give below a standard definition for $\mu$ and $\bar{\mu}$ which shows that they are model independent. We need a definition and some easy lemmas.

DEFINITION 3.6. For $A$ a sequence and $k, n$ natural numbers we let $r_{k}=$ the $2^{k}$ th root of $\frac{1}{2}$ and define

$$
\mu(n, k, A)=\frac{1}{n} \sum_{i}\left(r_{k}^{i} n-r_{k}^{i+1} n:\left[r_{k}^{i+1} n, r_{k}^{i} n\right] \cap A \neq 0\right) .
$$

The number $r_{k}$ is chosen so that the following lemma holds.

LEMMA 3.7. For a fixed $A$ and $n, \mu(n, k, A)$ is a decreasing function of $k$.

Proof. We note that $\mu(n, k, A)$ is $1 / n$ times the sum of the lengths of the intervals shown below which contain elements of $A$ :

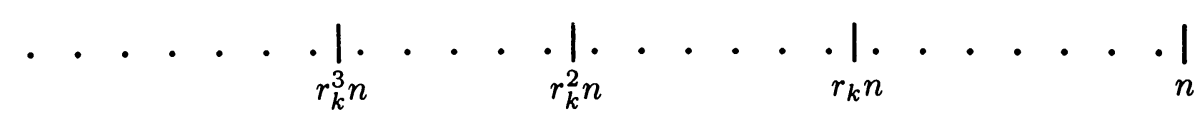

Since $r_{k+1}^{2}=r_{k}$ the partitions get strictly finer as $k$ increases.

We note that we have a symbol for $\mu$, so that it makes sense to write $\mu\left(z, k,{ }^{*} A\right)$ for $z \in{ }^{*} \mathbf{N}-\mathbf{N}$ and $k$ either finite or infinite.

LEMMA 3.8. For $z \in{ }^{*} \mathbf{N}-\mathbf{N}$ and $A$ any sequence of reals we have

$$
m\left(\operatorname{st}_{z}(A)\right)=\lim _{k \rightarrow \infty} \operatorname{st}\left(\mu\left(z, k,{ }^{*} A\right)\right)
$$

where $k$ runs through only finite values.

Proof. It is clear that for any finite $k m\left(\operatorname{st}_{z}(A)\right) \leq \operatorname{st}\left(\mu\left(z, k,{ }^{*} A\right)\right)$. Since $\operatorname{st}_{z}(A)$ is closed, its complement is a countable union of disjoint open intervals $\bigcup_{j=1}^{\infty} I_{j}$. Given $\varepsilon>0$ find $J$ so that $m\left(\bigcup_{j=J+1}^{\infty} I_{j}\right)<\varepsilon$. Now choose $k$ large enough so that

$$
m\left(\bigcup_{j=1}^{J} I_{j}-\bigcup\left\{\left[r_{k}^{i+1}, r_{k}^{i}\right]:\left[r_{k}^{i+1}, r_{k}^{i}\right] \subseteq I_{j} \text { for some } j=1, \ldots, J\right\}\right)<\varepsilon .
$$

Then $m\left(\operatorname{st}_{\boldsymbol{z}}(A)\right) \geq \mu\left(z, k,{ }^{*} A\right)-2 \varepsilon$, so that $m\left(\operatorname{st}_{\boldsymbol{z}}(A)\right)=\lim _{k \rightarrow \infty} \operatorname{st}\left(\mu\left(z, k,{ }^{*} A\right)\right)$.

LEMMA 3.9. For $z \in{ }^{*} \mathbf{N}-\mathbf{N}$ :

$$
\begin{array}{ll}
m\left(\operatorname{st}_{z}(A)\right) \leq \operatorname{st}\left(\mu\left(z, k,{ }^{*} A\right)\right) & \text { for } k \text { finite }, \\
m\left(\operatorname{st}_{z}(A)\right) \geq \operatorname{st}\left(\mu\left(z, k,{ }^{*} A\right)\right) & \text { for } k \text { infinite. }
\end{array}
$$

PROOF. The first assertion is clear. The second one follows from Lemmas 3.8 and 3.9.

THEOREM 3.10 .

$$
\begin{aligned}
& \underline{\mu}(A)=\inf _{k} \varliminf_{n} \mu(n, k, A), \\
& \bar{\mu}(A)=\inf _{k} \varlimsup_{n} \mu(n, k, A) .
\end{aligned}
$$

ProOF. (i) $\underline{\mu}(A) \leq \inf _{k} \underline{\lim } \mu(n, k, A)$. 
Let $k$ be any (standard) natural number. By transfer we can find $z \in{ }^{*} \mathbf{N}-\mathbf{N}$ with $\mu\left(z, k,{ }^{*} A\right)$ in the monad of $\underline{\lim } \mu(n, k, A)$. Since $k$ is finite Lemma 3.9 yields

$$
m\left(\operatorname{st}_{z}(A)\right) \leq \operatorname{st}\left(\mu\left(z, k,{ }^{*} A\right)\right)=\underline{\lim } \mu(n, k, A),
$$

so that $\underline{\mu}(A) \leq \underline{\lim } \mu(n, k, A)$.

(ii) $\mu(A) \geq \inf _{k} \underline{\lim } \mu(n, k, A)$.

Let $\bar{\varepsilon}>0$ and find $z \in{ }^{*} \mathbf{N}-\mathbf{N}$ with $\mu(A) \geq m\left(\right.$ st $\left._{z}(A)\right)-\varepsilon$. By Lemma 3.8 we can find a finite $k$ such that $\mu\left(z, k,{ }^{*} A\right) \leq m\left(\right.$ st $\left._{z}(A)\right)+\varepsilon$. We now have

$$
\underline{\mu}(A) \geq \mu\left(z, k,{ }^{*} A\right)-2 \varepsilon \geq \underline{\lim } \mu(n, k, A)-2 \varepsilon,
$$

so that $\underline{\mu}(A) \geq \inf _{k} \underline{\lim } \mu(n, k, A)$.

(iii) $\bar{\mu}(A) \leq \inf _{k} \varlimsup \overline{\lim } \mu(n, k, A)$.

Let $\varepsilon>0$ and find $z \in{ }^{*} \mathbf{N}-\mathbf{N}$ with $\bar{\mu}(A) \leq m\left(\operatorname{st}_{z}(A)\right)+\varepsilon$. Then $\bar{\mu}(A) \leq$ $\mu\left(z, k,{ }^{*} A\right)+\varepsilon$ for any finite $k$, and by transfer

$$
\bar{\mu}(A) \leq \varlimsup \overline{\lim } \mu(n, k, A)+2 \varepsilon,
$$

so that

$$
\bar{\mu}(A) \leq \inf _{k} \varlimsup \mu(n, k, A) .
$$

(iv) $\bar{\mu}(A) \geq \inf _{k} \varlimsup \overline{\lim } \mu(n, k, A)$.

Suppose, to the contrary, that there is an $\varepsilon>0$ so that for all finite $k \bar{\mu}(A)<$ $\varlimsup \mu(n, k, A)-\varepsilon$. Then by transfer there is an infinite $k$ and $z$ so that $\bar{\mu}(A)<$ $\mu\left(z, k,{ }^{*} A\right)-\varepsilon$. However $\bar{\mu}(A) \geq m\left(\operatorname{st}_{z}(A)\right) \geq \operatorname{st}\left(\mu\left(z, k,{ }^{*} A\right)\right)$ by Lemma 3.9 . Since $\varepsilon$ is standard and thus not infinitesimal this is a contradiction.

REMARK 3.11. The theorem above provides a standard definition for the density functions $\underline{\mu}$ and $\bar{\mu}$ showing that these functions are model independent.

The theorem and example below illustrate that $\bar{\mu}(A)$ is "attained" although $\underline{\mu}(A)$ need not be.

THEOREM 3.12. For any infinite sequence $A$ there is a $z \in{ }^{*} \mathbf{N}-\mathbf{N}$ such that $m\left(\operatorname{st}_{z}(A)\right)=\bar{\mu}(A)$.

PROOF. Since $\mu(n, k, A)$ is a decreasing function of $k$ for fixed $n$ and $A$ it is easy to see that we can find an infinite $k$ and $z$ with $\operatorname{st}\left(\mu\left(z, k,{ }^{*} A\right)\right)=\bar{\mu}(A)$. Then

$$
\bar{\mu}(A)=\operatorname{st}\left(\mu\left(z, k,{ }^{*} A\right)\right) \leq m\left(\operatorname{st}_{z}(A)\right) \leq \bar{\mu}(A) .
$$

The first inequality above is from Lemma 3.9 and the last one is by definition of $\bar{\mu}$. Thus $m\left(\operatorname{st}_{z}(A)\right)=\bar{\mu}(A)$.

EXAMPLE 3.13. There is a sequence $A$ with $\mu(A)=0$ even though $A$ has the interval property (so that for all $z \in{ }^{*} \mathbf{N}-\mathbf{N} m\left(\mathrm{st}_{z}(A)\right)>0$ ).

PROOF. Divide the interval $\left[10^{n-1}, 10^{n}\right]$ into $k^{2}$ equal sized subintervals, where $n-k$ is the nearest square number below $n$ (we could use any infinite sequence whose terms grow farther apart instead of the squares). Now put every $k$ th subinterval into $A$.

It is easy to see that for $k$ finite and $n$ infinite with $n-k$ a square and $z=10^{n}$ we have $m\left(\operatorname{st}_{z}(A)\right) \leq 2 / k$ so that $\underline{\mu}(A)=0$. Nevertheless $A$ has the interval property. To see this let $x$ be infinite and $z=10^{n}$ the largest power of 10 below $x$. Let $n-k$ 
be the largest square below $n$. If $k$ is finite then $\operatorname{st}_{z}(A)$ contains an interval of size $\left(1 / k^{2}\right)(.9)$ while an infinite $k$ makes st $_{z}(A)=$ all of $[0,1]$. Either interval is still an interval in st ${ }_{x}(A)$ since $z$ is not infinitesimal to $x$.

For a sequence of natural numbers we can compare the new density functions with the classical ones.

Proposition 3.14. $\underline{\mu}(A) \geq \underline{d}(A), \bar{\mu}(A) \geq \bar{d}(A)$.

PROOF. For any $n, k$ it is clear that $\mu(n, k, A) \geq A(n) / n$.

We now want to define a notion which is similar to that of "basis". For motivation we note the following simple facts.

Proposition 3.15. (i) A sequence $A$ of natural numbers is a basis iff for some $k \sigma(k A)=1$.

(ii) If the sequence $A$ contains the numbers 0 and 1 then $A$ is a basis iff for some $k \underline{d}(k A)=1$.

ProOF. (i) Clear.

(ii) If $A$ is a basis of order $k$ then $\underline{d}(k A)=1$.

If there is a $k$ so that $\underline{d}(k A)=1$ then, since 0 and 1 are in $A, \sigma(k A)>0$ and $A$ is a basis by Corollary 3.4.

Proposition 3.15 motivates the following definitions.

Definition 3.16. A sequence $A$ is called full iff $\underline{\mu}(A)=1$.

DEFinition 3.17. A sequence $A$ of reals is called a partial basis iff there is a $k$ so that $k A$ is full.

We note below some simple facts.

Proposition 3.18. The sequence $A=\left\langle a_{n}\right\rangle$ is full iff $\lim _{n \rightarrow \infty} a_{n+1} / a_{n}=1$.

PROOF. $\Rightarrow$ Suppose that $\lim _{n \rightarrow \infty} a_{n+1} / a_{n} \neq 1$. So there is an $\varepsilon>0$ with arbitrarily large $n$ for which $a_{n+1} \geq(1+\varepsilon) a_{n}$, and by transfer we may take $z=a_{n+1}$ where $n$ is infinite. Then $m\left(\operatorname{st}_{\boldsymbol{z}}(A)\right) \leq 1 /(1+\varepsilon)$ so that $\mu(A)<1$ and $A$ is not full. $[0,1]$.

$\Leftarrow$ If $\lim _{n \rightarrow \infty} a_{n+1} / a_{n}=1$ then for any infinite $z$ it is clear that $\operatorname{st}_{z}(A)=$

Proposition 3.19. The sequence $A$ is full iff for any infinite $z \operatorname{st}_{z}(A)=[0,1]$.

PROOF. Clear from Proposition 3.18.

It is important to note that $\underline{\mu}$ and $\bar{\mu}$, like $\underline{d}$ and $\bar{d}$, are asymptotic densities which would not change if the sequence $A$ were altered at finitely many points. Nevertheless the theorem below shows that in some ways $\underline{\mu}$ behaves more like $\sigma$ than like $\underline{d}$.

THEOREM 3.20 .

$$
\underline{\mu}(A+B) \geq \min \left\{\begin{array}{l}
\underline{\mu}(A)+\underline{\mu}(B), \\
1 .
\end{array}\right.
$$


PROOF. Let $z$ be any infinite element of ${ }^{*} \mathbf{N}$ and suppose that $m\left(\operatorname{st}_{z}(A+B)\right)<$ $\underline{\mu}(A)+\underline{\mu}(B) \leq 1$. For $k \in \mathbf{N}$ define

$$
\begin{aligned}
E_{1} & =\operatorname{st}_{z}(A), \\
E_{2} & =\operatorname{st}_{z}(B), \\
E_{3} & =\operatorname{st}_{z}(A+B), \\
U_{k} & =\bigcup_{n}\left\{\left[\frac{n}{k}, \frac{n+1}{k}\right]:\left[\frac{n-1}{k}, \frac{n+2}{k}\right] \cap E_{3}=0\right\} .
\end{aligned}
$$

$U_{k}$ is illustrated below.

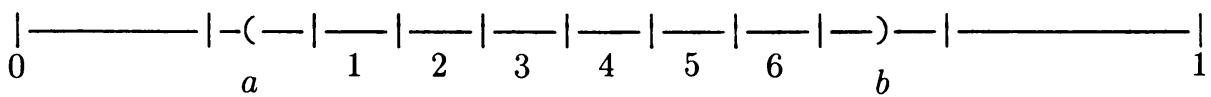

$(a, b)$ is an interval in $\tilde{E}_{3}$; the numbered intervals are of length $1 / k$. Intervals 2 through 5 are in $U_{k}$.

It is clear that $m\left(U_{k}\right) \rightarrow m\left(\tilde{E}_{3}\right)$, so we can find $k$ with

$$
m\left(U_{k}\right)>1-\underline{\mu}(A)=\underline{\mu}(B) .
$$

With $k$ now fixed we define two new finite sequences of length $k$ by

$$
\begin{array}{ll}
a_{i}=m & \text { iff }[m / k,(m+1) / k] \text { is the } i \text { th interval of length } 1 / k \\
& \text { to intersect } E_{1}, \\
b_{i}=m & \text { iff }[m / k,(m+1) / k] \text { is the } i \text { th interval of length } 1 / k \\
& \text { to intersect } E_{2} .
\end{array}
$$

So that $\sigma$ will be defined we will place all integers larger than $k$ in $\left\langle a_{i}\right\rangle$ and $\left\langle b_{i}\right\rangle$.

We note that

$$
\sigma\left(\left\langle a_{i}\right\rangle\right) \geq \underline{\mu}(A) \text { and } \sigma\left(\left\langle b_{i}\right\rangle\right) \geq \underline{\mu}(B) \text { since } m\left(\text { st }_{y}(A)\right) \geq \underline{\mu}(A)
$$

and $m\left(\operatorname{st}_{y}(B)\right) \geq \mu(B)$ for all infinite $y$.

We also note that if $[n / k,(n+1) / k]$ is in $U_{k}$ then there can be no $i, j \leq k$ with $a_{i}+b_{j}=n\left(\right.$ for then there would be an element of $E_{3}$ within $1 / k$ of $[n / k,(n+1) / k]$ ) so that

$$
m\left(U_{k}\right) \leq 1-\sigma\left(\left\langle a_{i}\right\rangle+\left\langle b_{i}\right\rangle\right) .
$$

But by Mann's Theorem (3.3),

$$
\sigma\left(\left\langle a_{i}\right\rangle+\left\langle b_{i}\right\rangle\right) \geq \sigma\left(\left\langle a_{i}\right\rangle\right)+\sigma\left(\left\langle b_{i}\right\rangle\right) \geq \underline{\mu}(A)+\underline{\mu}(B) \text { by }(2) .
$$

Inequalities (3) and (4) now yield $m\left(U_{k}\right) \leq 1-\underline{\mu}(A)-\underline{\mu}(B)$ which contradicts (1).

COROLlary 3.21. If $A$ is a sequence with $\underline{\mu}(A)>0$ then $A$ is a partial basis.

PROOF. Immediate from Theorem 3.20.

EXAMPLE 3.22. The analogous theorem to 3.3 and 3.20 is not true for $\underline{d}$. For example let $A=B=$ all multiples of 10 . Then

$$
\underline{d}(A)=\underline{d}(B)=\underline{d}(A+B)=1 / 10 .
$$

Even if both $A$ and $B$ contain the numbers 0 and 1 it is false. For example let

$$
A=B=\{\text { all numbers equal to } 0 \bmod 10 \text { or equal to } 1 \bmod 10\} \text {. }
$$


Then $\underline{d}(A)=\underline{d}(B)=\frac{1}{5}$ but $\underline{d}(A+B)=\frac{3}{10}$. (Note that $\sigma(A)=\frac{1}{9}$ and $\sigma(A+B)=\frac{2}{9}$.)

EXAMPLE 3.23. It is easy to find sequences $A$ with $\bar{\mu}(A)=0$ but $A$ a basis of order 2. We may, for example, let $A$ be all natural numbers which contain no 9 when written to the base 10 . Then $\operatorname{st}_{z}(A)$ is a Cantor type set of measure zero for any infinite $z$ (this is easiest to check for $z=10^{n}$ for some infinite $n$ ) so that $\bar{\mu}(A)=0$. Clearly every natural number can be written as a sum of two numbers in $A$.

We now ask for bounds on the thinness of a partial basis and the thickness of a nonpartial basis (in terms of the classical density functions). We know from Corollary 3.21 and Proposition 3.14 that if $\underline{d}(A)>0$ then $A$ is a partial basis. On the other hand it is shown in [Le] that any $e$-isolated sequence is not a partial basis. In particular any sequence $\left\langle\left[r^{n}\right]\right\rangle$ where $r>1$ is not a partial basis. Such sequences $A$ satisfy

$$
A(x) / \log x \leq C \quad \text { where } C \text { is constant. }
$$

Erdös and Schinzel [E] notice that no sequence which is so thin could be a partial basis and show that any partial basis $A$ must satisfy

$$
\varlimsup_{n} A(n) / \log n=\infty .
$$

The corollary below improves this result.

THEOREM 3.24. Let $A$ be a sequence and $C$ a constant in $\mathbf{N}$ such that for arbitrarily large $x$,

$$
A(x) / \log x \leq C .
$$

Then for any natural number $k, k A$ does not have the interval property.

ProOF. We show that for such an $A$ there is a $z \in{ }^{*} \mathbf{N}-\mathbf{N}$ so that $\operatorname{st}_{z}(A)$ is isolated. By Theorem $2.12 k A$ will then not have the interval property.

We first find a sequence $B$ such that $A \subseteq B, B(x) / \log x \geq 2 C$ but, $[B(x) / \log x]$ $=2 C$ for infinitely many $x \in \mathbf{N}$.

Let $x_{0}$ be such that $A(x) / \log x \leq C$ for $x>x_{0}$ and let $B$ be defined inductively as follows:

$$
B \cap x_{0}=A \cap x_{0} \cup\left\{\text { enough points }<x_{0} \text { so that }\left[B\left(x_{0}\right) / \log x_{0}\right]=2 C\right\} .
$$

We now place all succeeding integers of $A$ into $B$ as well as any $n$ for which $B(n-1) / \log n<2 C$ and it is clear that $B$ satisfies the desired properties.

By transfer there is an infinite $z \in{ }^{*} \mathbf{N}-\mathbf{N}$ so that ${ }^{*} B(z) / \log z=2 C$.

Let $y<z$ be noninfinitesimal to $z$, and write $r=z / y$, so that $r$ is finite in ${ }^{*} \mathbf{Q}$. Then

$$
{ }^{*} B(z)=2 C \log z=2 C \log (r y)=2 C \log r+2 C \log y .
$$

Since ${ }^{*} B(y) \geq 2 C \log y$ we have

$$
{ }^{*} B(z)-{ }^{*} B(y) \leq 2 C \log r,
$$

so that there are only finitely many elements of ${ }^{*} B$ between $y$ and $z$. This means that $\mathrm{st}_{\boldsymbol{z}}(B)$ (and hence $\mathrm{st}_{\boldsymbol{z}}(A)$ ) is isolated. 
COROllary 3.25. If $A$ is a partial basis then

$$
\lim _{n \rightarrow \infty} A(n) / \log n=\infty \text {. }
$$

ProOF. Immediate from Theorem 3.24.

The next example shows that Theorem 3.24 cannot be made stronger.

EXAMPLE 3.26. Let $f(n)$ be any function from $\mathbf{N}$ to $\mathbf{N}$ such that $\lim _{n \rightarrow \infty} f(n)$ $=\infty$. Then there is a sequence $A$ which is full but satisfies $A(n) / \log (n) \leq f(n)$ for all $n$.

PROOF. We first find $g(n) \leq f(n)$ which is nondecreasing and unbounded (in the obvious way). Now construct $A$ by subdividing every interval of the form $\left[2^{n}, 2^{n+1}\right]$ into $g(n)$ equal parts and placing all of the endpoints of the intervals into $A$. Then for $2^{n} \leq m \leq 2^{n+1}$ we have

$$
A(m) / \log m \leq A\left(z^{n+1}\right) / \log \left(z^{n}\right) \leq n g(n) / n \leq g(m) \leq f(m) .
$$

It is easy to see that $\lim _{n \rightarrow \infty} a_{n+1} / a_{n}=1$, so that $A$ is full.

At the other extreme we have the following example which shows that $A$ could have "nearly positive" density and yet not be a partial basis.

EXAMPLE 3.27. Let $f(n)$ be any nondecreasing function from $\mathbf{N}$ to $\mathbf{N}$ with $\lim _{n \rightarrow \infty} f(n)=\infty$. Then there is a sequence $A$ satisfying $A(n) \geq n / f(n)$ for all $n$ which is not a partial basis.

ProOF. Let $A$ be all positive integers between $2^{n}-2^{n+1} / f\left(2^{n}\right)$ and $2^{n}$. Then, for $2^{n} \leq m \leq 2^{n+1}$,

$$
A(m) \geq A\left(2^{n}\right) \geq 2^{n+1} / f\left(2^{n}\right) \geq m / f(m) .
$$

For any infinite $z \in{ }^{*} \mathrm{~N}, \mathrm{st}_{z}(A)=\mathrm{st}_{z}\left(\left\langle 2^{n}\right\rangle\right)$ so that $A$ is not a partial basis.

4. We now wish to translate some classical properties of Lebesgue measure into statements about sequences of natural numbers. Consequences of the Lebesgue density theorem seem especially suited for generating theorems which are difficult to prove without nonstandard methods. We start with a well-known theorem of Steinhaus.

THEOREM 4.1 (STEINHAUS). If $A$ and $B$ are sets of real numbers of positive measure then $A+B$ contains an interval.

Proof. Let $x$ be a point of density of $A$ and $y$ be a point of density of $B$. Choose $z$ so that for any $u$ between 0 and $z$ :

$$
m(A \cap[x, x+u])>\frac{1}{2} u \text { and } m(B \cap[y, y+u])>\frac{1}{2} u .
$$

Then for each $u$ in $[0, z]$ there must be a $t$ between 0 and $u$ so that $x+t \in A$ and $y+u-t \in B$. Thus $x+y+u$ is in $A+B$, so that $[x+y, x+y+z] \subseteq A+B$.

THEOREM 4.2. If $\underline{\mu}(A)>0$ and $\underline{\mu}(B)>0$ then $A+B$ has the interval property.

PROOF. This is immediate from Theorems 2.8 and 4.1 .

COROLlary 4.3. If $\underline{d}(A)>0$ and $\underline{d}(B)>0$ then $A+B$ has the interval property.

PROOF. This is clear from Proposition 3.14 and Theorem 4.2.

By the result of Merrill (Theorem 2.7) Theorem 4.2 and Corollary 4.3 should be particularly difficult to prove without nonstandard methods. However even the corollary below appears to be difficult. 
THEOREM 4.4. If $\bar{\mu}(A)>0$ and $\underline{\mu}(B)>0$ then neither $A+A$ nor $A+B$ is e-nowhere dense.

ProOF. By Theorem 4.1 there is a $z$ so that st $_{z}(A+A)$ and st $_{z}(A+B)$ contain intervals and thus $A+A$ and $A+B$ are not $e$-nowhere dense by Proposition 2.3.

COROllary 4.5. If $\bar{d}(A)>0$ and $\underline{d}(B)>0$ then neither $A+A$ nor $A+B$ is e-nowhere dense.

PROOF. This is clear from Proposition 3.14 and Theorem 4.4.

EXAMPLE 4.6. For any positive $r<1$ we can have $\underline{d}(A)=r$ with $A$ e-nowhere dense. In fact if $E \subseteq[0,1)$ is any closed nowhere dense set of positive measure we write

$$
\tilde{E}=\bigcup_{n=1}^{\infty}\left(r_{n}, s_{n}\right)
$$

and define $A$ by removing from every interval $\left[2^{n}, 2^{n+1}\right]$ all numbers between $2^{n}+$ $r_{i} \cdot 2^{n}$ and $2^{n}+s_{i} \cdot 2^{n}$ for $i=1, \ldots, n$. Then for any infinite $z \in{ }^{*} \mathbf{N} \operatorname{st}_{z}(A)$ contains a copy of $E$ in each interval $\left[\operatorname{st}\left(2^{n} / z\right), \operatorname{st}\left(2^{n+1} / z\right)\right]$ below $z$. Thus $\operatorname{st}_{z}(A)$ is always nowhere dense. Since $\sum_{n=1}^{\infty}\left(s_{n}-r_{n}\right)=1-m(E)$ it is clear that $\underline{d}(A)=$ $m(E)$. We now note that we could take $B$ to be an $e$-isolated but "nearly positive density" sequence as in Example 3.27 and $A+B$ is $e$-nowhere dense, showing that no hypothesis can be eliminated in results 4.2 through 4.5 .

Finally, Jan Mycielski asks if either corollary is sufficient to prove the classical Steinhaus result (Theorem 4.1). We show below that this is indeed the case, so that results 4.2 through 4.5 could be thought of as various "sequence versions" of this theorem.

THEOREM 4.7. Corollary 4.3 implies Theorem 4.1 and Corollary 4.5 implies Theorem 4.1.

PROOF. Suppose that $E_{1}$ and $E_{2}$ are sets of positive measure in $[0,1]$ such that $E_{1}+E_{2}$ contains no interval. We may assume that $E_{1}$ and $E_{2}$ are closed (if not take closed sets of positive measure contained in our original sets), so that $E_{1}+E_{2}$ is nowhere dense (closed and contains no interval). We now construct sequences $A$ and $B$ as in Example 4.6. That is, we write

$$
\tilde{E}_{1}=\bigcup_{n=1}^{\infty}\left(r_{n}, s_{n}\right) \text { and } \tilde{E}_{2}=\bigcup_{n=1}^{\infty}\left(u_{n}, v_{n}\right)
$$

$A$ is obtained by removing from every interval $\left[2^{n}, 2^{n+1}\right]$ all numbers between $2^{n}+r_{i}$. $2^{n}$ and $2^{n}+s_{i} \cdot 2^{n}$ for $i=1, \ldots, n$ and $B$ is obtained by removing from every interval $\left[2^{n}, 2^{n+1}\right]$ all numbers between $2^{n}+u_{i} \cdot 2^{n}$ and $2^{n}+v_{i} \cdot 2^{n}$. Then $\operatorname{st}_{z}(A)+\operatorname{st}_{z}(B)$ can be written as the countable union

$$
\bigcup_{n, m} \operatorname{st}_{z}(A) \cap\left[\operatorname{st}\left(2^{n} / z\right), \operatorname{st}\left(2^{n+1} / z\right)\right]+\operatorname{st}_{z}(B) \cap\left[\operatorname{st}\left(2^{m} / z\right), \operatorname{st}\left(2^{m+1} / z\right)\right] .
$$

Since each summand is a (translated and shrunk) copy of $E_{1}+a$ (translated and shrunk) copy of $E_{2}$ this union is meager and thus nowhere dense (since it is closed). This contradicts both Corollary 4.3 and Corollary 4.5. 
5. It is anticipated that many of the general techniques introduced here can be applied to known combinatorial problems. There are probably many more applications of the density theorem, for example, than those given in $\S 4$. Two very general questions then are:

QUESTION 5.1. What types of combinatorial problems can a nonstandard approach best be of help for? Presumably many questions in [EG], for example, might be attacked by these general methods.

QUESTION 5.2. Do many of the theorems involving basis and asymptotic or Schnirelmann density (e.g. those in [HR]) have interesting analogues involving the $\mu$-densities or partial bases?

Some more specific questions:

QUESTION 5.3. Suppose that $A_{i}$ limit-separates $B_{i}$ for $i=1, \ldots, N$ and that $\sum_{i=1}^{N} B_{i}$ is a partial basis. Is $\sum_{i=1}^{N} A_{i}$ a partial basis? Theorem 2.11 shows that $\sum_{i=1}^{N} A_{i}$ at least has the interval property. Even if between every two elements of $B_{i}$ there is an element of $A_{i}$ the conclusion is not clear. (The corresponding question with "basis" replacing "partial basis" is easily seen to be false.)

QUESTION 5.4. Are there easy conditions about a sequence $A$ which are equivalent to its being a partial basis?

QUESTION 5.5. For sequences of natural numbers, what additional conditions will ensure that a partial basis is, in fact, a basis? This question is undoubtedly very difficult. Thus, while it is clear that, for any $k$, the sequence $\left\langle n^{k}\right\rangle$ is a partial basis, the conjecture of Waring's that such a sequence is a basis was open for nearly 150 years before it was resolved in the affirmative by Hilbert.

\section{REFERENCES}

[E] P. Erdös, Personal communication.

[EG] P. Erdös and R. L. Graham, Old and new problems and results in combinatorial number theory, L'Enseignment Math., Université de Genève, 1980.

[HR] H. Halberstam and K. F. Roth, Sequences, Oxford Univ. Press, London, 1966.

[HKK] C. W. Henson, M. Kaufmann and H. J. Keisler, The strength of nonstandard methods in arithmetic, J. Symbolic Logic 49 (1984), 1039-1058.

[HM] J. Hirschfeld and M. Machover, Lectures on nonstandard analysis, Lecture Notes in Math., vol. 94, Springer-Verlag, Berlin and New York, 1969.

[K] G. Kreisel, Axiomatizations of nonstandard analysis that are conservative extensions of formal systems for classical standard analysis, Applications of Model Theory to Algebra, Analysis and Probability (W. A. J. Luxemburg, ed.), Holt, Rinehart and Winston, New York, 1969, pp. 93-106.

[Le] S. Leth, Sequences in countable nonstandard models of the natural numbers, (to appear in a special volume of Studia Logica (M. Szabo and M. Richter, eds.)).

[Lo] P. Loeb, An introduction to nonstandard analysis and hyperfinite probability theory, Probabilistic Analysis and Related Topics, Vol. 2 (A. J. Barucha-Reid, ed.), Academic Press, New York, 1979, pp. 105-142.

[M] J. Merrill, Some results in set theory and related fields, Doctoral thesis, Univ. of Wisconsin, 1986.

[R] A. Robinson, Nonstandard analysis, North-Holland, Amsterdam, 1966.

Department of Mathematics, UniVERsity of Wisconsin, Madison, Wisconsin 53706 